DOI: https://doi.org/10.11144/Javeriana.umed60-4.ssia

\title{
Signos semiológicos de la insuficiencia aórtica: apuntes para su historia
}

\section{Semiological Signs of Aortic Insufficiency: History Notes}

Recepción: 25 Marzo 2019 | Aceptación: 11 Junio 2019

\author{
Margarita Valencia Mejía \\ Residente de Medicina Interna, Facultad de Medicina, \\ Pontificia Universidad Javeriana, Bogotá, Colombia \\ Ángel Alberto García Peña ${ }^{a}$ \\ Profesor del Departamento de Medicina Interna, \\ Facultad de Medicina, Pontificia Universidad \\ Javeriana, Bogotá, Colombia
}

a Correspondencia: angel.garcia@javeriana.edu.co

Cómo citar: Valencia Mejía M, García Peña ÁA. Signos semiológicos de la insuficiencia aórtica: apuntes para su historia. Univ. Med. 2019;60 (4). htt ps://doi.org/10.11144/Javeriana.umed60-4.ssia

\section{RESUMEN}

La insuficiencia aórtica, referida como la incompetencia de la válvula para generar un adecuado cierre, constituye una de las valvulopatías con mayor riqueza semiológica. A lo largo de la historia, se ha descrito una gran variedad de signos periféricos, la mayoría de ellos epónimos de sus autores. El objetivo del presente artículo es hacer una revisión de la historia de los signos semiológicos más comunes y las contribuciones más relevantes de quienes los describieron.

Palabras clave

insuficiencia aórtica; historia; epónimos.

\section{ABSTRACT}

The aortic insufficiency is the inability of the valve to close properly, with a great amount of physical findings. The objective of this article is to make a review of the history and medical contributions of the authors who describe the principles signs.

Keywords

aortic valve insufficiency; history; eponyms.

\section{Introducción}

La insuficiencia aórtica, referida como la incompetencia de la válvula para generar un adecuado cierre, constituye una de las valvulopatías con mayor riqueza semiológica. A lo largo de la historia, se ha descrito una gran diversidad de signos periféricos, cuyo fundamento fisiopatológico es el mismo. A pesar del desarrollo tecnológico, el examen físico sigue siendo la principal herramienta para el diagnóstico de este tipo de valvulopatías, que posteriormente debe confirmarse y graduar la severidad mediante un ecocardiograma en cualquiera de sus modalidades. Ninguno de los hallazgos semiológicos puede precisar de forma independiente la información obtenida en 
un ecocardiograma. El objetivo del presente artículo es hacer una descripción de la vida y contribuciones a la medicina de los personajes que dieron el nombre a muchos de ellos.

Se presume que la primera descripción la realizó Abu Bakr Muhammad ibn Zakariya alRazi, cuyo nombre en latín es Rhazes (1). Fue un matemático, filósofo y médico persa de los más representativos de la medicina islámica a finales del siglo IX a. C. En uno de sus manuscritos se encuentra la descripción de un paciente que acudió por palpitaciones. Al examinarlo, Rhazes encontró un intenso latido ventricular izquierdo que jamás había presenciado y fuerte pulso braquial que incluso era visible (1). No tuvo el diagnóstico preciso de la enfermedad, pero su reseña es muy similar a la que años más tarde, sir Dominic Corrigan y sir Thomas Watson hicieron de este hallazgo semiológico.

\section{Sir Dominic Corrigan}

Nació el 2 de diciembre de 1802 en Dublín, Irlanda. Su padre era agricultor y comerciante de herramientas para la siembra (figura 19. Recibió su título de médico de la Universidad de Edimburgo, en 1825. Regresó años después a su país de origen donde empezó a trabajar como médico en el Jervis Street Hospital (2). Se destacó por ser un gran observador con sus pacientes. Publicó más de 100 artículos sobre diversos temas: desde discusiones sobre la fiebre tifoidea, la estructura del píloro, la escarlatina y la erupción sifilítica hasta la intoxicación por cobre. En su época se dio la conocida gran hambruna irlandesa (1845-1849), en la cual murieron más de un millón de habitantes por una plaga sobre los cultivos de papa y condiciones sociales difíciles para los irlandeses, explicadas en su gran mayoría por el dominio inglés. Fue un crítico fuerte de la situación y, en 1846, publicó un comunicado sobre la hambruna y la fiebre (3).

\section{Figura 1}

Retrato de sir Dominic Corrigan

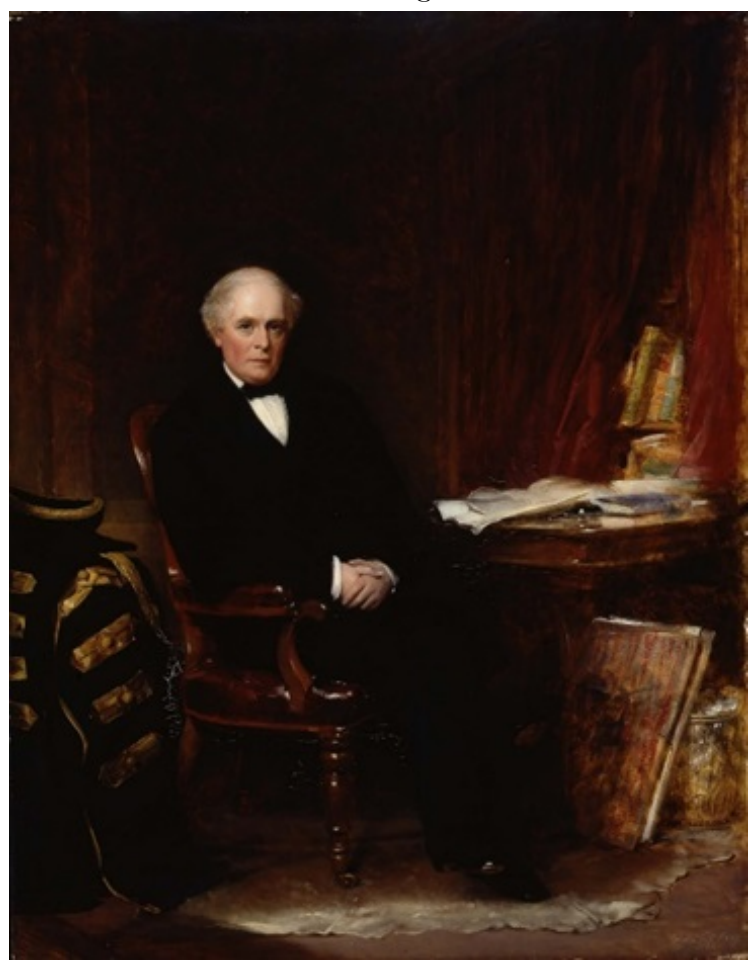

Fuente: cortesía de la

National Library of Medicine.

Tuvo importantes reconocimientos a lo largo de su vida. Fue médico honorario de la Universidad de Dublín (1849), trabajó como médico de la reina Victoria (1870) y se convirtió en vicerrector de la Universidad de Queens. Murió en 1880, al parecer, por un evento cerebrovascular (4). La descripción que sir Corrigan realizó inicialmente de los pulsos en la insuficiencia aórtica fue principalmente visual. Notó el rápido ascenso y colapso de las arterias del cuello en los pacientes con dicha valvulopatía.

Más adelante, se acuñó el término de pulso en martillo de agua (un artefacto de vidrio lleno de agua al vacío) por los escritos de sir Thomas Watson, quien comparó el golpe producido por el agua al chocar con uno de los extremos del tubo a la palpación del pulso radial (4). 


\section{Sir Austin Flint}

Nació en Petersham, Massachusetts, en 1812. Su padre, Edward Flint, también fue médico de la guerra de independencia de Estados Unidos. Obtuvo su título de médico de la Universidad de Harvard, en 1833. Años más tarde, contrajo matrimonio con Annie Skillings y después del nacimiento de su primer hijo se mudó a Búfalo, Nueva York. En 1845, fundó la revista médica de Búfalo, y dos años después el colegio médico del mismo nombre (5). Tuvo una importante influencia en la literatura médica de su época. Las enfermedades infecciosas constituían la mayoría de los temas de sus publicaciones, lo que reflejaba la prevalencia de estas en Estados Unidos en aquel tiempo (figura 2).

\section{Figura 2}

Austin Flint

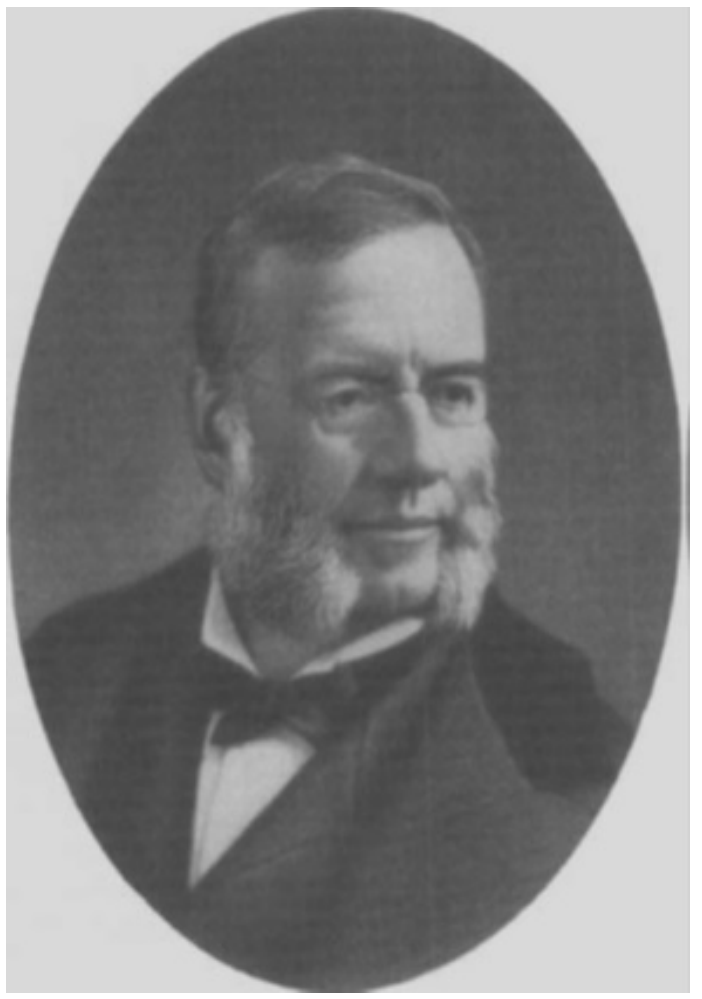

Fuente: tomado de Physicians and Surgeons of The United States por Charles Robson.

Entre sus artículos se destacan "Practical Treatise on the Diagnosis, Pathology and Treatment of Disease of the Heart", y el libro de 1886: A Treatise on the Principles and Practice of Medicine (5). En ellos se evidenciaba la importancia que su autor daba a la semiología para el diagnóstico de las enfermedades cardiacas y describió un soplo en pacientes con insuficiencia aórtica que recibe su nombre. El soplo de Austin Flint es uno mesodiastólico que se puede auscultar en el ápice y que mejora al encontrarse el paciente en decúbito lateral izquierdo y con la campana del estetoscopio. Inicialmente, se interpretó como una estenosis mitral funcional, originada por el aumento del volumen telediastólico del ventrículo izquierdo; sin embargo, años más tarde surgieron otras teorías. Entre estas se incluía la sobreposición del jet de insuficiencia aórtica con el flujo anterógrado mitral, las vibraciones en el endocardio del ventrículo izquierdo por la insuficiencia o el choque del flujo con la valva anterior de la válvula mitral (5). No existe una teoría clara en la actualidad; se cree que la causa puede ser multifactorial.

\section{Paul Louis Duroziez}

Nació el 8 de enero de 1826, en París, Francia. Se graduó como médico de la Escuela de Medicina de París, en 1853 (2). Aun siendo estudiante, ganó el Premio Corvisart, por su monografía sobre los propiedades fisiológicas y terapéuticas de los digitálicos. En 1856, fue nombrado jefe del L'Hôpital de la Charité, en la misma ciudad. Sus principales intereses de estudio eran las enfermedades cardiacas y entre sus publicaciones se encuentra la descripción de la estenosis mitral, 1861 (2). Creía que el corazón podía ser descrito en dos mitades: una mitad izquierda, la cual consideraba la parte masculina, regular y calmada, y una mitad derecha, femenina, desordenada y nerviosa (6). En 1891, publicó su obra maestra titulada Traité clinique des maladies $d u$ coeur. Su importancia dentro de la historia de la valvulopatía aórtica radica en la descripción de un doble soplo, sistólico y diastólico, intermitente sobre la arteria femoral. Este se reproduce comprimiendo la arteria con el estetoscopio y se pensó en su momento era explicado por la entrada de la sangre en la extremidad inferior 
durante la sístole y el flujo retrogrado durante la diástole (7). La fisiopatología de este signo ha generado controversia desde su descripción.

En 1933, Blumbgart y Ernstene (8) concluyeron que el componente en diástole podría también producirse por el flujo continuo en pacientes con tirotoxicosis y anemia por vasodilatación periférica, no siendo específico de la insuficiencia aórtica y recomendaban sumergir la extremidad en agua fría para diferenciarlo. Años más tarde, en 1965, a través de angiografías en animales y humanos con insuficiencia aórtica se corroboró la fisiopatología inicialmente descrita (7). No incluyeron pacientes con el soplo de Duroziez, sin insuficiencia aórtica en su investigación; sin embargo, hasta hoy en día se mantiene lo descrito por su autor. Duroziez decía que en este tipo de pacientes el soplo tiende a ser continuo, en vez de intermitente (6) y de mayor intensidad al levantar el estetoscopio (7).

\section{Alfred de Musset}

El signo de Musset es uno de los epónimos en cardiología que recibe su nombre por el paciente y no por quién lo describió. Nació el 11 de diciembre de 1810, en París, Francia. Estudió en Henry IV College y se graduó en 1827. Estudió pintura y literatura, y se convirtió en un importante poeta y dramaturgo de la época (2). Entre sus poesías se destacan "Rolla" (1833) y "Les Nuits" (1836), y su novela La confession d 'un enfant du siécle (1836). Al parecer, su vida estuvo llena de excesos. En los últimos años se le diagnosticó una insuficiencia aórtica, se cree secundaria a sífilis. Falleció a los 46 años. Fue su hermano Paul, quien en su biografía describió el signo semiológico que recibe su nombre (9). En 1842, mientras desayunaban con su madre, notó el balanceo que realizaba con su cabeza de forma involuntaria en reposo. El término médico fue descrito por Armand Delpeuch, en 1900 (10).

\section{Sir Leonard Erskine Hill}

Nació el 2 de junio de 1866, en Tottenham, Inglaterra (figura 3). Estudió en el Colegio
Médico de Londres, donde se destacó por sus reconocimientos en fisiología y anatomía. Fue tanto su interés en el área que fue aprendiz del famoso fisiólogo Edward H. Schafer (11). Sus primeras investigaciones se concentraban en la circulación, inicialmente en la cerebral, por lo que en 1896 publicó "The Physiology and Pathology of the Cerebral Circulation”. Trabajó junto con H. L. Barnard en el desarrollo de un instrumento para la medición de la presión arterial; sin embargo, en 1896 Scipione RivaRocci se le adelantó con el esfingomanómetro. En 1897, con Barnard, realizan modificaciones a este adicionando el manómetro de aguja (11). En 1914, se convirtió en el director del Departamento de Fisiología Aplicada en el National Institute of Medical Research.

\section{Figura 3}

Sir Leonard Erskine Hill (1866-1952)

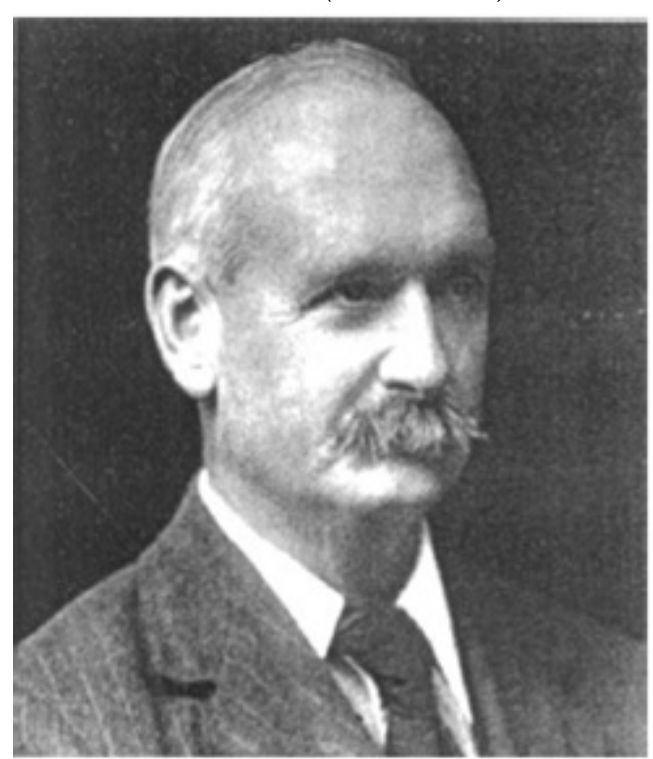

Fuente: tomado del obituario de Fellows of the Royal Society of London (1952-1953;21:8).

$\mathrm{Su}$ contribución a la insuficiencia aórtica la realizo en 1909. Junto con el doctor W. Holtzmann, describió una diferencia de presión arterial al comparar la medición en arteria braquial contra la poplítea o tibial en pacientes con esta valvulopatía en decúbito supino. Utilizando un esfingomanómetro, la presión sistólica estaba determinada por la desaparición del pulso radial o tibial posterior. Investigaciones 
posteriores confirmaron que no existe una diferencia en la medición intrarterial de dichas zonas. Se cree que es un artefacto de la medición indirecta (12). Frank et al. (13) propusieron una diferencia igual o mayor a $20 \mathrm{~mm} \mathrm{Hg}$ para considerar positivo el signo.

\section{Otto Heinrich Enoch Becker}

Nació el 3 de mayo de 1828 en Ratzeburg, Alemania. Se graduó de oftalmología de la Universidad de Viena y se convirtió en catedrático de la Universidad de Heidelberg. Dentro de sus áreas de investigación se encontraban la circulación sanguínea en la retina (14). Describió las pulsaciones que se dan en la arteria retiniana en los pacientes con insuficiencia aórtica.

\section{Heinrich Irenaeus Quincke}

Nació en 1842, en Fráncfort del Óder, Alemania. Estudió medicina en la Universidad Heidelberg, Wurzburgo y Berlín, donde recibió clases de famosos personajes de la época entre los que se encontraban Rudolf Virchow, Rudolf Albert von Kolilker, Johannes Peter Müller, Hermann von Helmholtz. A los 30 años, se convirtió en profesor de medicina interna en Bern. Describió el angioedema y fue el primero en proponer el drenaje quirúrgico para los pacientes con abscesos pulmonares y fue la primera persona en publicar la poiquilocitosis en un paciente con anemia perniciosa (14). Sin embargo, su contribución más importante fue la realización de la primera punción lumbar como método diagnóstico y terapéutico. Teniendo en cuenta los estudios descritos sobre fisiología de la circulación cerebroespinal, consideró que los niños con hidrocefalia se podrían beneficiar del drenaje de líquido cefalorraquídeo (14). Describió las pulsaciones que se podían observar en el lecho ungueal de pacientes con insuficiencia aórtica.

\section{Friederich von Müller}

Laringólogo alemán. Inicialmente estudió ciencias naturales en Múnich, y años más tarde, medicina, en dicha ciudad, y en Wurzburgo (14). En Bonn, trabajó como profesor de propedéutica clínica y laringología. Junto a Hamman, recibió el signo semiológico que recibe el nombre de este último, que se puede encontrar en pacientes con neumomediastino (14). En 1889, describió la pulsación sistólica de la úvula acompañado de eritema y edema del paladar y amígdalas.

\section{Signo de Lincoln}

Este epónimo, al igual que el signo de Musset, recibe su nombre por el paciente en quien se documentó: al décimo sexto presidente de Estados Unidos: Abraham Lincoln. Todo surge gracias a Noah Brooks, un periodista y amigo muy cercano del mandatario (15). En alguna ocasión, le mostró una foto de Lincoln, en la que se podía ver al presidente sentado con una pierna cruzada sobre la otra, pero curiosamente la primera se veía borrosa (figura 4). Extrañado, admitió no haberse movido. Al intentar reproducir el episodio, observaron el movimiento oscilante de su extremidad. En 1972, Schwartz (15) relacionó estos hallazgos con la hipótesis que Lincoln padecía de síndrome de Marfan, y con ello, insuficiencia aórtica. El movimiento de la pierna no era más que la transmisión de la velocidad y presión del flujo aórtico, evidente al hacer compresión sobre la arteria poplítea, sumada a la hiperlaxitud de sus articulaciones. 


\section{Figura 4}

\section{Abraham Lincoln}

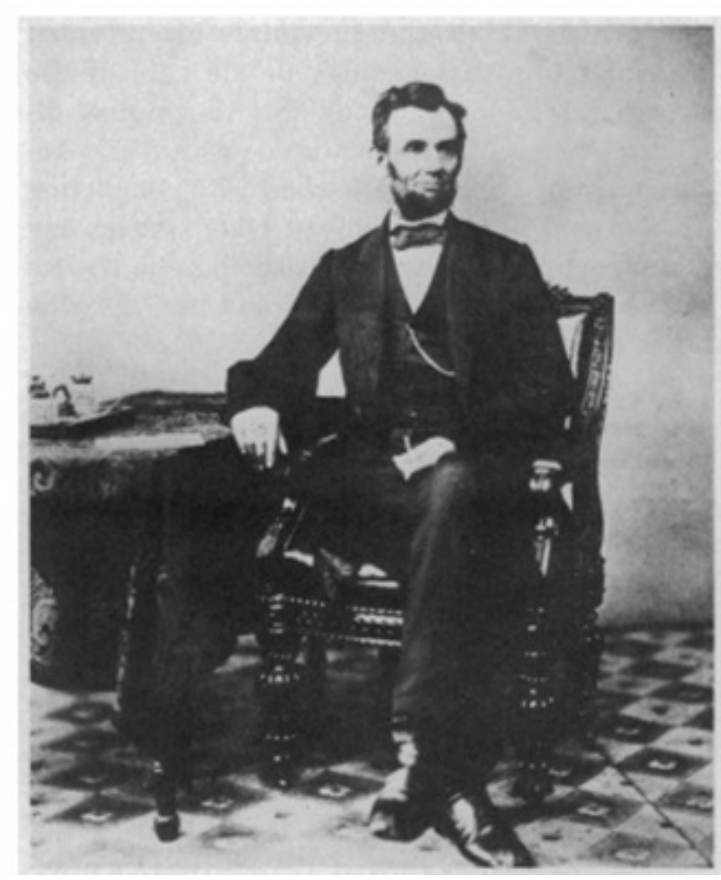

Fuente: tomado de Lincoln en fotografías por C. Hamilton y L. Ostendorf.

Tabla 1

Resumen

\begin{tabular}{|c|c|c|c|}
\hline Signo & Autor & Descripción & Fisiopatología \\
\hline $\begin{array}{l}\text { Pulso de Corrigan } \\
\text { Pulso martillo de } \\
\text { agua }\end{array}$ & $\begin{array}{l}\text { Dominc Corrigan, } \\
\text { Irlanda (1802-1880) } \\
\text { Thomas Watson, } \\
\text { Inglaterra (1792- } \\
\text { 1882) }\end{array}$ & $\begin{array}{l}\text { Rápido ascenso y } \\
\text { colapso de las } \\
\text { arterias carótida. } \\
\text { Compara el golpe } \\
\text { producido por el } \\
\text { agua al chocar con el } \\
\text { extremo de este } \\
\text { artefacto con el } \\
\text { pulso radial. }\end{array}$ & $\begin{array}{l}\text { Rápido descenso de } \\
\text { la presión aortica en } \\
\text { la fase diastólica } \\
\text { secundario a la } \\
\text { incompetencia } \\
\text { valvular que genera } \\
\text { amplia diferencia de } \\
\text { presiones** }\end{array}$ \\
\hline $\begin{array}{l}\text { Soplo de Austin } \\
\text { Flint }\end{array}$ & $\begin{array}{l}\text { Austin Flint, } \\
\text { Estados Unidos } \\
\text { (1812-1886) }\end{array}$ & $\begin{array}{l}\text { Soplo } \\
\text { mesodiastólico que } \\
\text { se puede auscultar } \\
\text { en el ápice y que } \\
\text { mejora al } \\
\text { encontrarse el } \\
\text { paciente en decúbito } \\
\text { lateral izquierdo y } \\
\text { con la campana del } \\
\text { estetoscopio. } \\
\end{array}$ & $\begin{array}{l}\text { Se cree que es } \\
\text { multifactorial (véase } \\
\text { el artículo) }\end{array}$ \\
\hline Signo de Duroziez & $\begin{array}{l}\text { Paul Louis Duroziez, } \\
\text { Francia (1826-1897) }\end{array}$ & $\begin{array}{l}\text { Doble soplo, } \\
\text { sistólico y diastólico, } \\
\text { intermitente sobre la } \\
\text { arteria femoral. Este } \\
\text { se reproduce } \\
\text { comprimiendo la } \\
\text { arteria con el } \\
\text { esteoscopio. }\end{array}$ & $\begin{array}{l}\text { Generado por la } \\
\text { entrada de la sangre } \\
\text { en la extremidad } \\
\text { inferior durante la } \\
\text { sístole y el flujo } \\
\text { retrógrado durante } \\
\text { la diástole. } \\
\text { En tirotoxicosis y } \\
\text { anemia el soplo } \\
\text { tiende a ser continuo } \\
\text { y de mayor } \\
\text { intensidad al } \\
\text { levantar el } \\
\text { estetoscopio. }\end{array}$ \\
\hline
\end{tabular}

Tabla 1 (cont.)

Resumen

\begin{tabular}{|l|l|l|l|}
\hline Signo de Musset & $\begin{array}{l}\text { Armand Delpeuch, } \\
\text { Francia (1853-1901), } \\
\text { inspirado en el } \\
\text { famoso dramaturgo } \\
\text { y poeta Alfred de } \\
\text { Musset }\end{array}$ & $\begin{array}{l}\text { Balanceo de la } \\
\text { cabeza en reposo. }\end{array}$ & $\begin{array}{l}\text { Véase primera celda } \\
\text { de esta columna }\end{array}$ \\
\hline Signo de Hill & $\begin{array}{l}\text { Leonard Erskine } \\
\text { Hill, Inglaterra } \\
\text { (1866-1952) }\end{array}$ & $\begin{array}{l}\text { Describió una } \\
\text { diferencia de presión } \\
\text { arterial al comparar } \\
\text { la medición en } \\
\text { arteria braquial } \\
\text { versus la poplitea o } \\
\text { tibial diferencia de } \\
\text { 20 mm Hg se } \\
\text { considera } \\
\text { significativa. }\end{array}$ & $\begin{array}{l}\text { Se cree que es un } \\
\text { artefacto de la } \\
\text { medición indirecta. }\end{array}$ \\
\hline Signo de Becker & $\begin{array}{l}\text { Otto Heinrich Enoch } \\
\text { Becker, Alemania } \\
\text { (1828-1890) }\end{array}$ & $\begin{array}{l}\text { Pulsaciones en } \\
\text { arteria retininana. }\end{array}$ & $\begin{array}{l}\text { Véase primera celda } \\
\text { de esta columna }\end{array}$ \\
\hline Signo de Quincke & $\begin{array}{l}\text { Heinrich Irenaeus } \\
\text { Quincke, Alemania } \\
\text { (1842-1922) }\end{array}$ & $\begin{array}{l}\text { Pulsaciones en } \\
\text { lechos ungueales. }\end{array}$ & $\begin{array}{l}\text { Véase primera celda } \\
\text { de esta columna }\end{array}$ \\
\hline Signo de Müller & $\begin{array}{l}\text { Friederich von } \\
\text { Mler, Alemania } \\
\text { (1858-1941) }\end{array}$ & $\begin{array}{l}\text { Pulsacion sistólica } \\
\text { sobre la úvula } \\
\text { acompañado de } \\
\text { eritema y edema del } \\
\text { paladar y las } \\
\text { amígdalas. }\end{array}$ & $\begin{array}{l}\text { Véase primera celda } \\
\text { de esta columna }\end{array}$ \\
\hline Signo de Lincoln & $\begin{array}{l}\text { Harold Swharz, } \\
\text { Estados Unidos }\end{array}$ & $\begin{array}{l}\text { Movimiento } \\
\text { oscilante de la piema } \\
\text { al estar cruzada } \\
\text { sobre la otra en } \\
\text { posición sentado. }\end{array}$ & $\begin{array}{l}\text { Resultado de la } \\
\text { transmisión de la } \\
\text { velocidad y presión } \\
\text { del flujo aórtico } \\
\text { evidente al hacer } \\
\text { compresión sobre la } \\
\text { arteria poplitea. }\end{array}$ \\
\hline
\end{tabular}

\section{Discusión}

La insuficiencia aórtica es la valvulopatía con mayor riqueza semiológica $y$, al mismo tiempo, reflejo de la historia de la medicina, y su desarrollo a lo largo de los siglos XIX y XX. Con este artículo se resalta la vida y contribuciones de sus autores. Previamente se han realizado revisiones similares $(2,12,16)$; sin embargo, vale la pena mencionar el trabajo realizado por Babu et al. (12). Ellos llevaron a cabo una revisión sistemática de la literatura con el objetivo de determinar de la sensibilidad y especificidad de cada signo. Solo el signo de Hill, de Duroziez, el pulso de Corrigan y el soplo de Austin Flint tenían estudios suficientes. Los trabajos tenían pocos pacientes y se utilizaban diferentes métodos en un mismo estudio para definir insuficiencia aórtica, lo cual podría generar sesgos. La sensibilidad para cada signo manejaba rangos demasiado amplios que no podían ser concluyentes; sin embargo, un estudio demostró que el grado de severidad se correlacionaba con el signo de Hill, lo que sugería podría ser el más específico. En ninguna de las revisiones se habló de Rhazes o el signo de Lincoln. 
Con el desarrollo de múltiples modalidades de diagnóstico cardiovascular y el interés en investigación, la evaluación de características operativas de cada uno de los signos o de alguna utilidad específica entró en desuso, dado el pobre aporte, cuando se compara con información de la ecocardiografía, por lo que han sido relegados a aspectos puramente académicos o con fines educativos.

En el momento de examinar a un paciente con sospecha de valvulopatía, estos signos deben ser buscados de forma meticulosa en un examen físico, ya que nos pueden orientar un diagnóstico y tratamiento. De igual forma, conocer la historia de quienes describieron sus principales signos semiológicos nos ayuda a facilitar su aprendizaje.

\section{Referencias}

1. Dalfardi B, Mahmoudi Nezhad GS, Ghanizadeh A. Rhazes' description of a case with aortic regurgitation. Int J Cardiol. 2014;172(1):e147-8. https://d oi.org/10.1016/j.ijcard.2013.12.096.

2. Nader JN. Eponyms in cardiology and cardiovascular surgery. s. 1.: Infinity Publishing; 2012.

3. Sir Dominic John Corrigan. JAMA. 1962;180(5):406-7. https://doi.org/10. 1001/jama.1962.03050180052015.

4. Stone J. Sir Dominic John Corrigan. Clin Cardiol. 1986;9:403-6.

5. Fye WB. Austin Flint, 1812-1886. Clin Cardiol. 1989;12:476-7.

6. Berry D. History of cardiology: Paul Louis Duroziez, M. Circulation. 2006;28;114(22):f188.

7. Rowe GG, Afonso S, Castillo CA, McKenna DH. The mechanism of the production of Duroziez's murmur. N Engl J Med. 1965;272:1207-10.

8. Blumgart HL, Erstene AC. Two mechanisms in the production of Duroziez's sign: their diagnostic significance and a clinical test for differentiating between them. J Am Med Assoc. 1933;100:173-7.

9. Mehta NJ, Khan IA. Original descriptions of the classic signs of aortic valve insufficiency. J Emerg Med. 2003;24(1):69-72.

10. Delpeuch A. Musset's sign: a rhythmic bobbing of the head in aortic cases. Presse Med. 1900;8:237-8.

11. Hill AB, Hill B. The life of Sir Leonard Erskine Hill FRS (1866-1952). Proc R Soc Med. 1968;61(3):307-16.

12. Babu AN, Kymes SM, Carpenter Fryer SM. Eponyms and the diagnosis of aortic regurgitation: what says the evidence? Ann Intern Med. 2003;138(9):736-42.

13. Frank MJ, Casanegra P, Miglori AJ, Levinson GE. The clinical evaluation of aortic regurgitation. Arch Intern Med. 1965;116:357-65.

14. Mangioni S. Physical diagnosis secrets. 2nd ed. Philadelphia: Mosby; 2008.

15. Schwartz H. Abraham Lincoln and aortic insufficiency - The declining health of the president (Clinical Note). Calif Med. 1972;116:82-4.

16. Mehta N, Khan I. Original descriptions of the classic signs of aortic valve insufficiency. J Emerg Med. 2003;24(1):69-72. 